\title{
ATM kinase enables the functional axis of YAP, PML and p53 to ameliorate loss of Werner protein-mediated oncogenic senescence
}

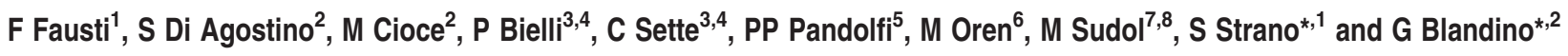

\begin{abstract}
Werner syndrome (WS) results from dysfunction of the WRN protein, and is associated with premature aging and early death. Here we report that loss of WRN function elicits accumulation of the Yes-associated protein (YAP protein), a major effector of the Hippo tumor suppressor pathway, both experimentally and in WS-derived fibroblasts. YAP upregulation correlates with slower cell proliferation and accelerated senescence, which are partially mediated by the formation of a complex between YAP and the PML protein, whose activity promotes p53 activation. The ATM kinase is necessary for YAP and PML accumulation in WRN-depleted cells. Notably, the depletion of either YAP or PML partially impairs the induction of senescence following WRN loss. Altogether, our findings reveal that loss of WRN activity triggers the activation of an ATM-YAP-PML-p53 axis, thereby accelerating cellular senescence. The latter has features of SASP (senescence-associated secretory phenotype), whose protumorigenic properties are potentiated by YAP, PML and p53 depletion.
\end{abstract}

Cell Death and Differentiation (2013) 20, 1498-1509; doi:10.1038/cdd.2013.101; published online 9 August 2013

The RecQ protein family is a highly conserved group of helicases with multiple roles ranging from DNA recombination, replication and repair to transcription. ${ }^{1-4}$ Human $R e c Q$ helicase include WRN (Werner), BLM (Bloom), RECQ4, RECQ1 and RECQ5 proteins. The alteration of RecQ helicases is associated with genetic recessive disorders. Among the latters, the Werner syndrome (WS) is characterized by the loss of helicase and exonuclease activities of the WRN protein ${ }^{5}$; WS is clinically characterized by premature aging, ${ }^{6}$ and by early death $(\leq 50$ years), due to cardiovascular diseases and cancer. ${ }^{7,8}$ The spectrum of tumors exhibited by WS patients consists of soft tissue and bone sarcomas. ${ }^{6}$ Despite intense clinical and molecular studies of WS pathology, the molecular mechanisms underlying the clinical progression of the WS patients remain only partially characterized. Here we report that small interfering RNA (siRNA)-mediated knockdown of the WRN protein in HCT116 cells results in a 2- to 10-fold accumulation of the Yes-associated protein (YAP) protein, followed by accelerated senescence of the targeted cells. This takes place in fibroblasts derived from WS patients as well. YAP is a WW domaincontaining transcriptional coactivator, ${ }^{9}$ reported to associate with various transcription factors regulating functionally opposite pathways, ranging from DNA damage-mediated apoptosis to oncogenic transformation. ${ }^{10,11}$ We have previously shown that $P M L$ gene is a transcriptional target of the protein complex p73/YAP. ${ }^{12,13}$ We have also found that PML protein binds to $Y A P$, protects it from degradation and reinforces the proapoptotic activity of the protein complex p73/YAP in response to DNA-damaging agents. ${ }^{11,12}$ The accumulation of YAP in the WRN K/D cells triggers the formation of a YAP-PML complex, whose activity impinges on the pro-senescent functions of p53. The ATM kinase activity is required for the accumulation of $\mathrm{YAP}$ and $\mathrm{PML}$ as well as for the stabilization of p53 and its phosphorylation at Ser15 following WRN knockdown. The YAP-PML-p53 axis may contribute to the cancer-prone features of WS patients. In fact, we show that the acquisition of a SASP phenotype (senescence-associated secretory phenotype), a cellular event known to exert protumorigenic effect via paracrine signaling to adjacent cells, ${ }^{14,15}$ is also a feature of WRN K/D senescent cells. Notably, reduced expression of YAP, PML and p53 proteins contributes to the establishment of SASP features in WRN K/D cells.

\section{Results}

Loss of WRN expression causes YAP protein accumulation. We found that the transient knocking down

\footnotetext{
${ }^{1}$ Molecular Chemoprevention Group, Molecular Medicine Area, Regina Elena National Cancer Institute, Rome, Italy; ${ }^{2}$ Translational Oncogenomic Unit, Molecular Medicine Area, Regina Elena National Cancer Institute, Rome, Italy; ${ }^{3}$ Department of Public Health and Cell Biology, University of Rome Tor Vergata, Rome, Italy; ${ }^{4}$ Laboratory of Neuroembryology, IRCSS Fondazione Santa Lucia, Rome, Italy; ${ }^{5}$ Cancer Genetics Program, Department of Medicine and Pathology, Beth Israel Deaconess Cancer Center, Beth Israel Deaconess Medical Center, Harvard Medical School, Boston, MA, USA; ${ }^{6}$ Department of Molecular Cell Biology, Weizmann Institute of Science, Rehovot, Israel; ${ }^{7}$ Weis Center for Research, Geisinger Clinic, Danville, PA, USA and ${ }^{8}$ Mount Sinai School of Medicine, New York, NY, USA ${ }^{*}$ Corresponding author: S Strano, Molecular Chemoprevention Group, Molecular Medicine Area, Regina Elena National Cancer Institute, Via Elio Chianesi, 53, Rome 00143, Italy. Tel: + 39065266 2974; Fax: + 39065266 5530; E-mail: strano@ ifo.it

or G Blandino, Translational Oncogenomic Unit, Molecular Medicine Area, Regina Elena National Cancer Institute, Via Elio Chianesi, 53, Rome 00143, Italy. Tel: + 39065266 2911; Fax: + 39065266 5530; E-mail: blandino@ifo.it

Keywords: Werner syndrome; cellular senescence; Yes-associated protein (YAP); ATM kinase

Abbreviations: WS, Werner syndrome; WRN, Werner syndrome protein; YAP, Yes-associated protein; PML, Promyelocytic Leukemia protein; SASP, Senescenceassociated secretory phenotype; SA- $\beta$-Gal, Senescence-associated $\beta$-galactosidase; BrdU, 5-Bromo-2'-deoxy-uridine

Received 05.2.13; revised 11.6.13; accepted 02.7.13; Edited by T Mak; published online 09.8.13
} 
of WRN expression in HCT116 cells caused YAP protein accumulation (2 to 10-fold over controls) (Figure 1a; Supplementary Figures $1 \mathrm{a}$ and $\mathrm{b}$; data not shown) which correlated with decreased proliferation and induction of p21 $1^{\text {waf1 }}$ (Figures $1 \mathrm{~b}$ and $\mathrm{c}$ ). The same was observed in stably K/D cells (shWRN-HCT116 cells), where we found that the accumulation of YAP, PML, p21 and phospho-p53 (Figure 1d) strongly correlated with the induction of senescence, as evidenced by high numbers of senescenceassociated beta-galactosidase (SA- $\beta$-Gal)-positive cells (Figure 1e; Supplementary Figures 1c-f). The accumulation of YAP, PML, p21 and phospho-p53 correlated with intense SA- $\beta$-Gal HCT116-positive cells undergoing low dosage of chemotherapeutic treatment-induced senescence (Supplementary Figures $2 \mathrm{a}-\mathrm{c}$ ). ${ }^{16,17}$ In addition, YAP levels were increased (1.8-folds) in senescence-prone fibroblasts derived from WS patients (Figure 1f) characterized by increased PML, p21 and p16INK4a mRNA levels, as well as decreased PTEN mRNA as compared with fibroblasts from healthy donors (supplementary Figure 3). siRNAmediated depletion of WRN slowed down cell proliferation and accelerated senescence of $\mathrm{WRN}^{+/+}$primary human skin fibroblasts (GM08402) (Figures 1g-i). This correlated with induced accumulation of YAP (Figure 1h). Thus, our HCT116-based cellular model (shWRN-HCT116 cells) closely recapitulated most aspects of the WS fibroblasts. Interestingly, ectopic expression of YAP (as well as of PML or p53) inhibited cell proliferation and promoted senescence of $\mathrm{WRN}^{+1+}$ normal primary fibroblasts (GM08402) (Supplementary Figures $4 \mathrm{a}-\mathrm{c}$ ). Altogether, this prompted us to investigate how the accumulation of YAP may impinge on the phenotype induced by the loss of WRN.

YAP contributes to senescence of WRN-deficient cells. We found that knocking down of YAP expression significantly impaired cellular senescence of pro-senescent shWRN-HCT116 cells (Figure 2a). This was accompanied by attenuated induction of PML, p21, p53 and of Ser15phosphorylated p53 (Figure 2a). At the same time, siRNAmediated depletion of PML in shWRN-HCT116 cells prevented senescence by diminishing p53 phosphorylation at Ser15 and induction of p21 waf1 protein (Figure 2b). Similar to that observed by knocking down of YAP or PML, the siRNA-mediated p53 deletion slowed down cellular senescence of shWRN-HCT116 cells and compromised the induction of p21 (Figure $2 \mathrm{c}$ ). This shares similarity with PMLregulated p53 activation observed in oncogene-infected, senescent cells. ${ }^{18}$ To detail the contribution of YAP, PML and p53 to the premature senescence of WRN-deficient cells, we depleted human WRN ${ }^{-1-}$ fibroblasts (AG03141) of those factors by siRNAs (Figures 2d-f; Supplementary Figure 5). This showed that depletion of YAP diminished PML, p53 and p21 ${ }^{\text {waf1 }}$ protein accumulation (Figures $2 \mathrm{dl}-\mathrm{IV}$ ). Similarly, PML depletion reduced p53 and p2 $1^{\text {waf1 }}$ levels (Figures 2dl-IV). Notably, siRNA-mediated knockdown of p53 impaired p21 $1^{\text {waf1 }}$ induction but did not affect YAP and $P M L$ accumulation, implying that their induction occurred upstream to p53 activation in the senescent cells (Figures 2dl-IV). Depletion of each of the three factors increased the fraction of BrdU-positive cells (as compared with control siRNA transfected cells), whereas the knocking down of wt-p53 severely impaired SA- $\beta$-Gal staining as well (Figures $2 \mathrm{e}$ and $\mathrm{f}$ ). This is compatible with YAP being part of a functional axis that includes PML and p53 in pro-senescent WRN K/D cells.

The YAP-PML-p53 functional axis promotes senescence of WRN-deficient cells. Pulse-chase experiments revealed that the half-life of YAP was slightly prolonged, whereas those of PML and p53 proteins were extended in shWRNHCT116 cells compared with control cells (Figures $3 a-c$ ). In addition, the transcripts of $Y A P, P M L$ and p53 genes were significantly increased in WRN-depleted cells (Supplementary Figures $6 \mathrm{a}-\mathrm{c}$ ), $P<0.05$ and enriched in the polysomal fraction (Figures $3 d-f$ ) as opposed to the non polysomal RNA fraction (Supplementary Figures 6d-f). We used HEK293 cells expressing -inducible YAP protein ${ }^{19}$ as positive control for the latter experiments (Supplementary Figures $6 \mathrm{~g}-\mathrm{j}$ ). Next, we assessed whether the increase of YAP, PML and p53 proteins in WRN K/D cells resulted in physical interactions. We proved the presence of a YAP-PML protein complex in siWRN-HCT116 cells (Figures $4 a$ and $b$ ). We also observed increased recruitment of $\mathrm{YAP}, \mathrm{PML}$ and $\mathrm{p} 53$ proteins to $p 21^{\text {waf1 }}$ regulatory regions enriched for hyper-acetylated histone-H4 (Figure 4c; Supplementary Figures $7 \mathrm{a}-\mathrm{-c}$ ), suggesting that the observed complexes were transcriptionally competent. Of note, YAP and PML proteins, whose physical interaction was not augmented in WRN K/D cells (Figure 4b), were concomitantly enriched onto p21 promoter (Figure 4d). Collectively these findings indicated that (i) YAP accumulation precedes PML-regulated p53 activation and the induction of the senescence program and that (ii) the contribution of YAP to induction of senescence upon loss of WRN may impinge on PML protein activity, enforcing p53 pro-senescence function(s).

\section{ATM, but not p38MAPK, is required for activation of the} YAP-PML-p53 functional axis upon loss of WRN activity. ATM kinase and p38MAPK are major regulators of p53 in response to DNA damage through phosphorylation of its ser15 residue. ${ }^{20,21}$ Therefore, we assessed their involvement in the p53 phosphorylation in WRN K/D senescent cells. Thus, we treated CTRL and WRN K/D cells (shGFP- and shWRN-HCT116 cells) with caffeine at a concentration known to specifically inhibit ATM $(5 \mathrm{mM}){ }^{22}$ or with SB202190, a selective inhibitor of p38 MAPK. Western blotting showed that both caffeine and SB202190 reduced p53 Ser15 phosphorylation and p21 waf1 induction in WRN K/D cells (Figure $5 a$ ), with caffeine only affecting the increase of YAP and PML (Figure 5a). Indirect immunofluorescence confirmed the different effect of the two drugs with regard to the nuclear accumulation of YAP, PML and p53 in the WRN $\mathrm{K} / \mathrm{D}$ cells (Figures $5 \mathrm{~b}-\mathrm{g}$ ). We confirmed the specificity of both caffeine and SB202190 toward ATM (Ser-1981 residue) and p38MAPK, respectively (Figures $5 \mathrm{~h}-\mathrm{j}$ ) by indirect immunofluorescence. ATM Ser-1981 phosphorylation was also evidenced in HCT116 cells undergoing low dosage of chemotherapeutic treatment-induced senescence (Supplementary Figures $8 \mathrm{a}$ and b). Next we explored 
a

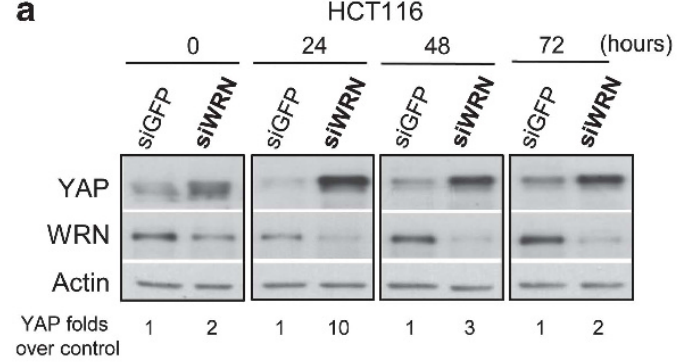

over contro

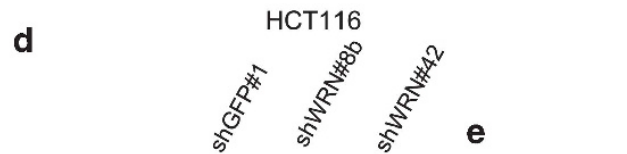

b

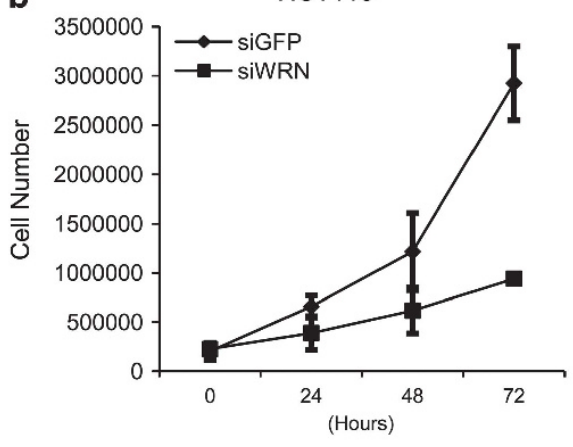

HCT116

21 protein levels

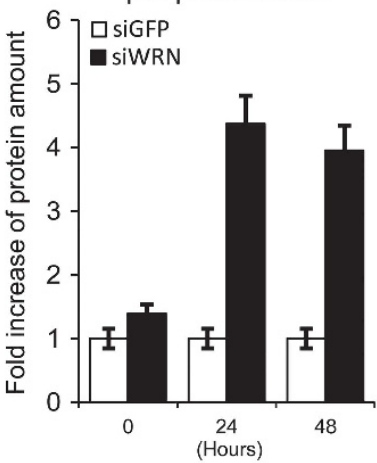

HCT116

shGFP\#1 shWRN\#42
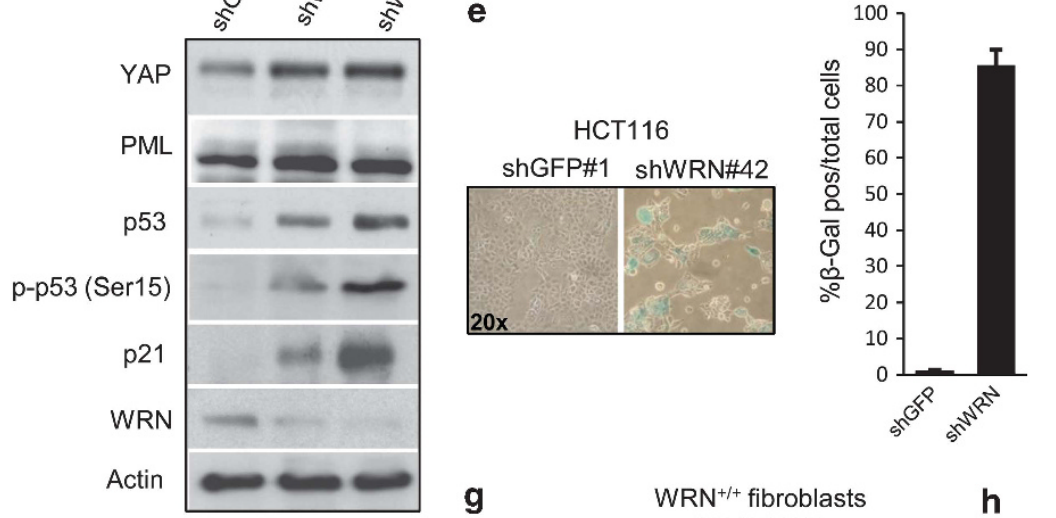

f
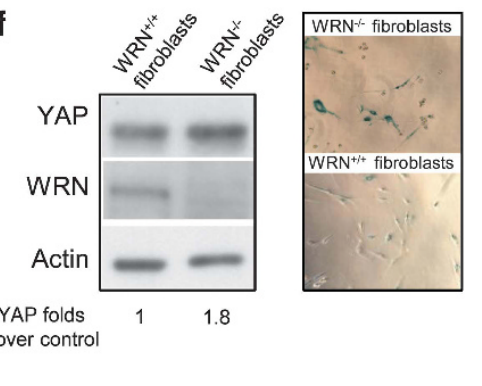

g

$\mathrm{WRN}^{+/+}$fibroblasts

(GM08402)

\begin{tabular}{|c|c|c|c|}
\hline $\begin{array}{c}\text { YAP folds } \\
\text { over control }\end{array}$ & 1 & 3 & 3.5 \\
\hline $\begin{array}{c}\text { PML folds } \\
\text { over control }\end{array}$ & 1 & 1,7 & 1,8 \\
\hline $\begin{array}{c}\text { p53 folds } \\
\text { over control }\end{array}$ & 1 & 3,8 & 9.0 \\
\hline $\begin{array}{c}\text { p-p53(Ser15) } \\
\text { folds } \\
\text { over control }\end{array}$ & 1 & 4,8 & 13 \\
\hline $\begin{array}{c}\text { p21 folds } \\
\text { over control }\end{array}$ & 1 & 5.0 & 19.0 \\
\hline
\end{tabular}

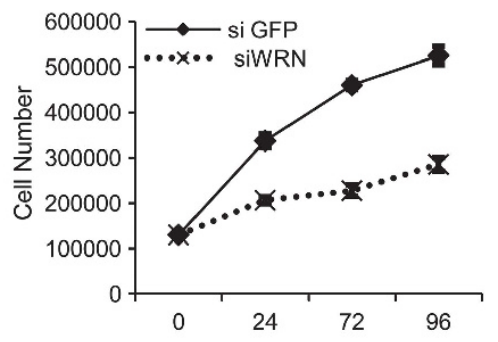

h

WRN $^{+/+}$fibroblasts (GM08402)

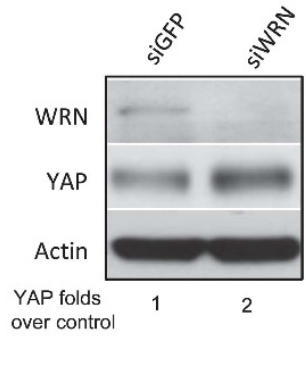

i $\mathrm{WRN}^{+/+}$fibroblasts

(GM08402)

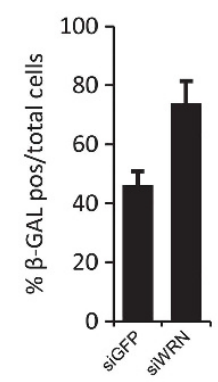

Figure 1 Silencing of WRN stabilizes YAP protein levels. (a) Western blotting analysis of WCL (whole-cell lysate) of HCT116 cells transfected with siRNA targeting WRN and GFP (as control), collected at the indicated time points and probed with the indicated antibodies. The 0 time point $(0 \mathrm{~h})$ was the starting point from the transfection wash, after overnight (about $16 \mathrm{~h}$ ) siRNAs incubation. (b) Proliferation of HCT116 transfected as from (a), evaluated by trypan blue staining, at the indicated times. Data show mean + S.D. of three independent cell counts. (c) Expression of p21 protein evaluated from HCT116 transfected as from (a): densitometric analysis. Histograms show mean + S.D. values of three independent experiments. (d) Upper panel: western blotting analysis for the expression of the indicated gene products from HCT116 cells stably knocked-down for WRN (shRNA) and for GFP, as control (shWRN-HCT116 and shGFP-HCT116). Different clones were derived for both shGFP-HCT116 and shWRN-HCT116 by limit dilution of transfected cells (as indicated for shGFP-HCT116 clone no. 1 and shWRN-HCT116 clone no. 8b and no. 42). Lower panel: densitometric analysis of the expression of the indicated gene products in the stable shGFP- and shWRN-HCT116 clones as from upper panel. (e) Left panel: SA- $\beta$-gal staining of HCT116 cells as from (d). Representative images of clones shGFP-HCT116 clone no. 1 and shWRN-HCT116 clone no. 42. Right panel: histograms show mean + S.D. values of three independent experiments. (f) Left panel: western Blotting analysis of YAP levels from WRN ${ }^{+1+}$ (GM08402) and WRN ${ }^{-1-}$ (AG03141) fibroblasts. Right panel: SA- $\beta$-gal staining of cells as from left panel. (g) Proliferation of WRN ${ }^{+1+}$ fibroblasts (GM08402) transfected with siRNA against WRN and GFP (as control), evaluated by trypan blue staining. Data show mean + S.D. of three independent cell counts. (h) Western Blotting analysis of WCL derived from WRN ${ }^{+1+}$ fibroblasts as from $(\mathbf{g})$, probed with the indicated antibodies (i). Histograms show mean + S.D. values of three independent experiments as from (g)

whether YAP and ATM may also physically interact. Coimmunoprecipitation experiments performed in $\mathrm{H} 1299$ expressing EGFP-YAP and FLAG-ATM revealed physical interaction (Figure 6a upper panel, lanes 1 and 4), with their binding involving the phosphorylation of YAP at ser/thr residues (Figure 6a upper panel, lanes 1 and 4). In line with this, caffeine treatment reduced ATM auto-phosphorylation and YAP phosphorylation (Figure 6a upper panel, lanes and 4) thus severely hampering the stability of the YAP-ATM complex (Figure 6a, upper panel, lane 3). Interestingly, treatment with the SB202190 MAPK inhibitor did not have effect on the YAP-ATM complex (Figure 6a upper panel, lanes 2 and 4). Altogether, this suggests physical and functional interactions between the ATM kinase and the YAP-PML-p53 axis in WRN K/D senescent cells. To deepen the previous observations, we assessed the effect 
a

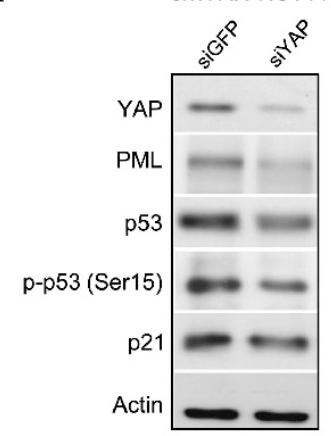

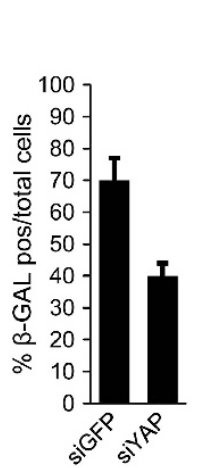

b

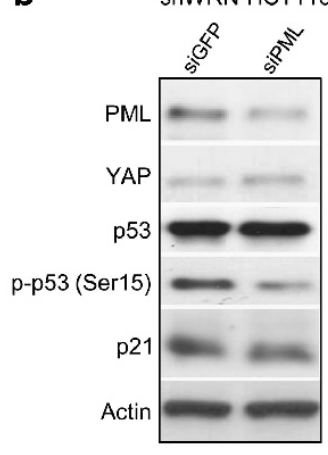

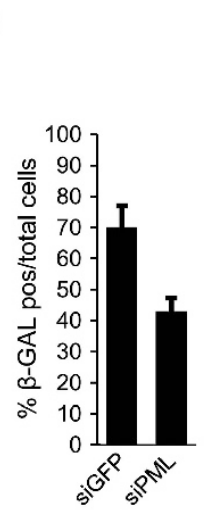

c

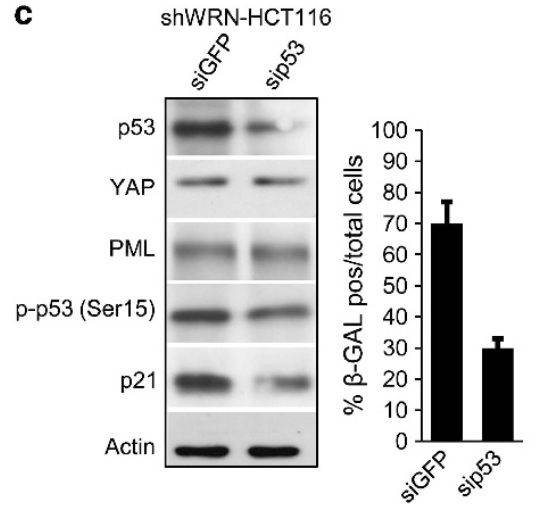

d

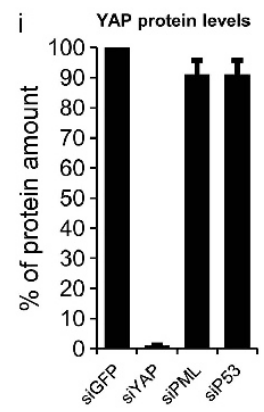

WRN $^{-1-}$ fibroblasts (AG03141) e
WRN-1- fibroblasts
(AG03141) $\mathbf{f}$

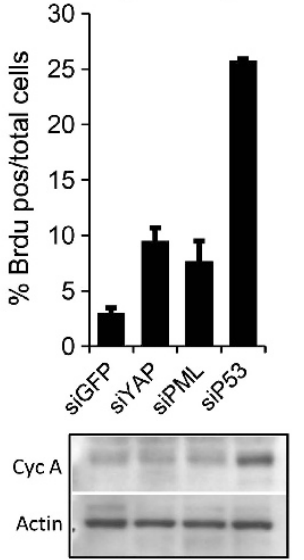

P53 protein levels iv p21 protein levels
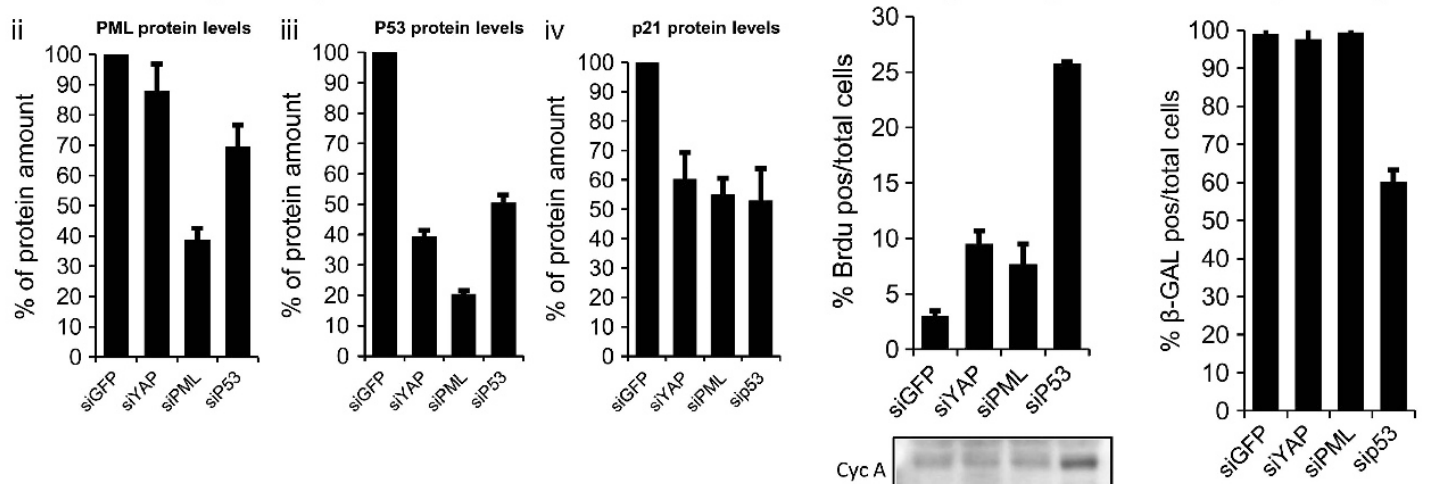

Figure 2 YAP, PML and p53 contribute to senescence in WRN K/D cells. (a-c) YAP, PML and p53 K/D in shWRN-HCT116 cells. Left panels: western Blotting analysis for the expression of the indicated gene products from shWRN-HCT116 transfected with siRNAs targeting either YAP (a) or PML (b) or p53 (c) or GFP (as control) and probed with indicated antibodies. Right panels: SA- $\beta$-gal staining of cells as from left panels. Histograms show mean + S.D. values of three independent experiments of shWRN-HCT116 transfected as from $(\mathbf{a}-\mathbf{c})$. Interfered shWRN-HCT116 cells were collected for protein levels and analyzed for SA- $\beta$-Gal staining $96 \mathrm{~h}$ (4 days) after siRNAs transfection. (d-f) YAP, PML and p53 KID in WS fibroblasts. (d I-IV) Densitometric analysis for the expression of YAP (d I), PML (d II), p53 (d III) and p21 (d IV) from WS fibroblasts (AG01341) transfected with siRNA targeting either YAP, PML, p53 or GFP (as control) (normalized to actin content). Histograms show mean + S.D. values of three independent experiments. (e) Upper panel: BrdU incorporation of cells as from (d). Cells were subjected to a $4 \mathrm{~h}$ Brdu pulse. Histograms show mean + S.D. values of three independent experiments. Lower panel: WCL from cells as from (d) probed with the indicated antibodies. f SA- $\beta$-gal staining of cells as from (d). Histograms show mean + S.D. values of three independent experiments. Interfered WS fibroblasts were collected for protein levels, analyzed for SA- $\beta$-Gal and Brdu staining $192 \mathrm{~h}$ (8 days) after siRNAs transfection

of WRN K/D in ATM-deficient cells (GM16666 fibroblasts) or in the same cells reconstituted with FLAG-ATM (GM16667 fibroblasts) (Figure 6b). As expected, we found that the accumulation of YAP, PML and phospho-p53 was observed only in the ATM-reconstituted cells depleted of WRN by siRNA (Figure 6b). Further, we found that ectopic expression of a kinase-defective ATM mutant but not of ATM wt in WRN $\mathrm{K} / \mathrm{D}$ cells prevented the increase of YAP, PML nuclear levels as well as Ser 15 phosphorylation of p53 and induction of p21 waf1 (Figures $6 \mathrm{c}-\mathrm{e}$ ). Notably, this resulted in reduced numbers of SA- $\beta$-Gal-positive WRN K/D cells (Figure 6f). Altogether, our findings suggest that ATM is a critical regulator of the YAP-PML-p53 functional axis during the senescence induced by the loss of WRN. We also provide evidence that other kinases such as p38MAPK can contribute to the accumulation of phospho-p53 in WRN $\mathrm{K} / \mathrm{D}$ cells.

WRN K/D mediated senescence resembles SASP. Senescent cells release a plethora of cytokines and pro-mitogenic factors and this process is called SASP. ${ }^{14,15}$ Interestingly, the induction of SASP by oncogenic stimuli and/or DNA damage can be a mechanism of tumor progression due to paracrine signaling. ${ }^{14,23,24}$ The analysis of the expression of key players of DNA damage repair following the activation of DNA damage response signaling such as NBS1, Ku70 and RAD17 transcripts, was unchanged along the senescence of both human $\mathrm{WRN}^{-1-}$ fibroblasts (AG03141) and shWRN-HCT116 cells when compared with their respective control cells (Supplementary Figures 9a-f). Instead, DNA-damaging treatment $(7.5 \mu \mathrm{g} / \mathrm{ml}$ of cisplatin) caused a significant increase of NBS1, ku70 and RAD17 expression in HCT116 cells (Supplementary Figures 9g-i). Given the increased frequency of tumors in progeric WS patients, ${ }^{6}$ we investigated whether the senescence induced by the loss of WRN has features of SASP and whether this contributes protumorigenic properties to adjacent cells. We therefore assessed the levels of 77 different cytokines and growth factors/receptors in the conditioned medium (CM) of shWRN-HCT116 and control cells (Supplementary 


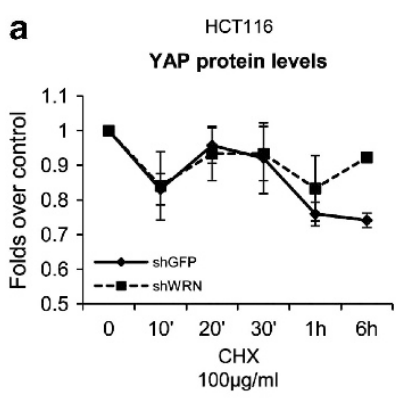

d
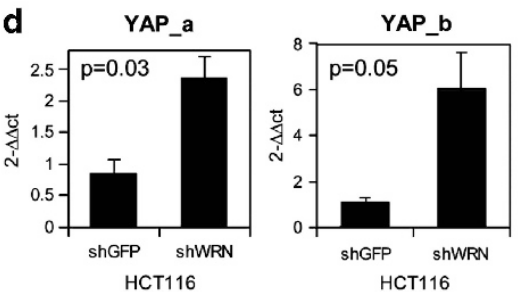

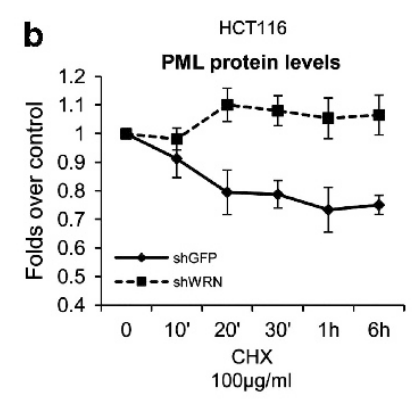

e

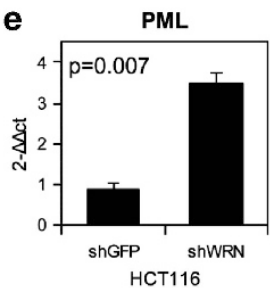

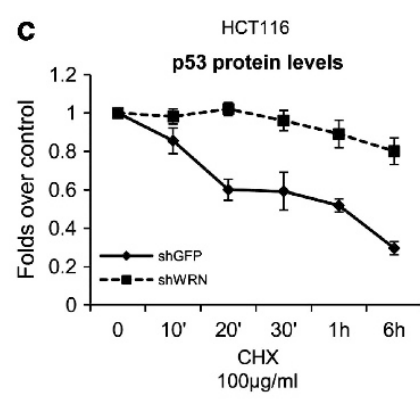

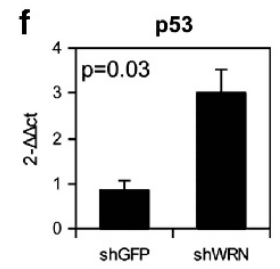

HCT116

Figure 3 Translation mechanism is involved in increased levels of YAP, PML and p53 in WRN K/D cells. (a-c) Densitometric analysis of endogenous levels of YAP (a), PML (b) and p53 (c) in shWRN-HCT116 cells and shGFP-, as control, upon cycloheximide $100 \mu \mathrm{M}$ treatment and collected at the indicated times. (d) Q-PCR analysis of mRNA levels of YAP polysomal fraction from shWRN-HCT116 cells and shGFP-, as control, evaluated using two different sets of primers (YAP_a and YAP_b) that amplify upstream and downstream regions of YAP transcript. Histograms show mean + S.D. values of three independent experiments. (e and f). Q-PCR analyses of mRNA level of polysomal fraction of PML (e) and p53 (f) from shWRN-HCT116 cells and shGFP-, as control. Histograms show mean + S.D. values of three independent experiments. All primers sequences are included in the Supplementary Figure 6 and in the Supplementary informations file

Table 1). This revealed that senescent, WRN K/D cells showed a profile of secreted cytokines and growth factors significantly different from their wt counterparts, with 40 out of 77 factors being differentially represented the $\mathrm{CM}$ of shWRN-HCT116 cells $(P<0.05)$ (Figure 7a). Among the latters, we found FGF-1, FGF-2, IFN $\gamma$, IGFI, IL-1 $\alpha$, IL-6, Inhibin alpha, ICAM1, MMP-11/(stromelysin-3) and MMP13/ (collagenese-3). These factors are known to be released by senescent cells upon stress stimuli such as DNA damage, ${ }^{15}$ and to be protumorigenic. ${ }^{15,23,24}$ Beside this, we unexpectedly found a number of proteins not known to be secreted and differentially enriched in the examined conditioned media, such as BCL2, BCL-X and BCL-XL. Those factors can be a part of exosome vesicles secreted by cancer cells, and capable of delivering antiapoptotic signals. ${ }^{25}$ In fact, the type of buffer used in the assay allows the extraction of exosome components (unpublished observations). We separately tested the levels of secreted interleukin-8 (IL-8) in the conditioned media from the shWRN-HCT116 and control cells (Figure $7 \mathrm{~b}$ right panel). IL-8 has been previously shown by us and others to be a prominent factor secreted by fibroblasts $^{15}$ and mesothelioma cells undergoing SASP ${ }^{24}$ (please note that IL-8 expression was not represented in the array illustrated in Figure 7a). This revealed that IL-8 protein levels were significantly higher in media of shWRN-HCT116 cells (as compared with control counterparts) and this matched with increased mRNA levels as assessed by qRTPCR (Figure 7b left panel). Moreover, this was the same in WS fibroblasts (as opposed to healthy fibroblasts), again reinforcing the similarity between our HCT116 model and the patient-derived cultures (Figure 7c). Increased protein levels of IL-8 were also found in HCT116 cells undergoing low dosage of chemotherapeutic treatment-induced senescence (Supplementary Figure 10). Next, we evaluated the levels of
IL-8 in the CM from shWRN-HCT116 cells transfected with YAP, PML or p53 targeting siRNAs (as opposed to siRNAGFP controls). We found that, although with some variability, silencing of each of the three factors induced higher levels of secreted IL-8 protein, with YAP siRNA having the major effect (Figure 7d). To test whether the different spectrum of secreted proteins in WRN K/D cells would also correlate with a different effect on the protumorigenic features of naïve, recipient cells, we evaluated the proliferative capacity of the untransformed $\mathrm{WRN}^{+/+}$fibroblasts (AG03141) and HFF-1 (human foreskin fibroblasts) exposed to conditioned media from the shWRN-HCT116 and control cells (Figures 8a and b). This revealed that the CM from WRN K/D cells was consistently capable of triggering an increased proliferation of both $\mathrm{WRN}^{+1+}$ and HFF-1 cells, as compared with control medium (Figures $8 a$ and $b$, left panels). This paired with an increase of the protein levels of both cell proliferation markers (Ki-67) and cell cycle regulators (cyclin A and E) (Figures $8 \mathrm{a}$ and $\mathrm{b}$, right panels). Next, we evaluated whether the same medium could enhance the protumorigenic properties of transformed epithelial breast cancer cells (MCF-7). The medium harvested from WRN K/D cells increased both proliferation and clonogenic ability of the exposed MCF7cells (as compared with medium from control cells) (Figure 8c, left and right panels). Altogether these findings show that the senescence induced by WRN K/D has SASPlike features and in fact may promote protumorigenic properties in adjacent cells and that YAP, PML and p53 affect the SASP-like features of senescent WRN K/D cells.

\section{Discussion}

WS is a human progeric syndrome that exhibits accelerated aging and, in most cases, premature death owing to 


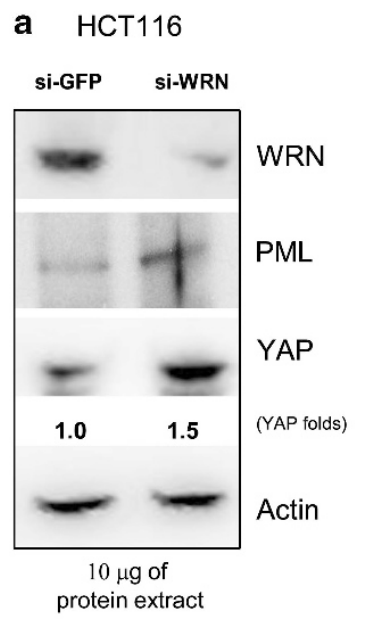

\section{b} HCT116

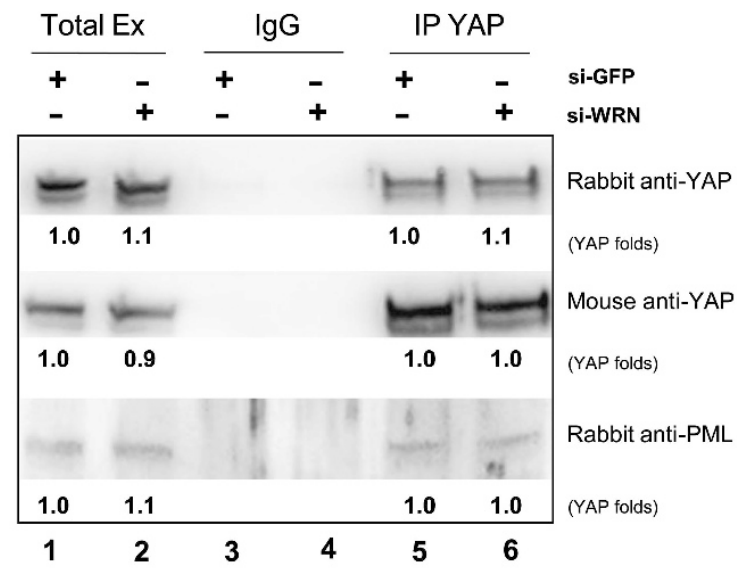

c

ChIP

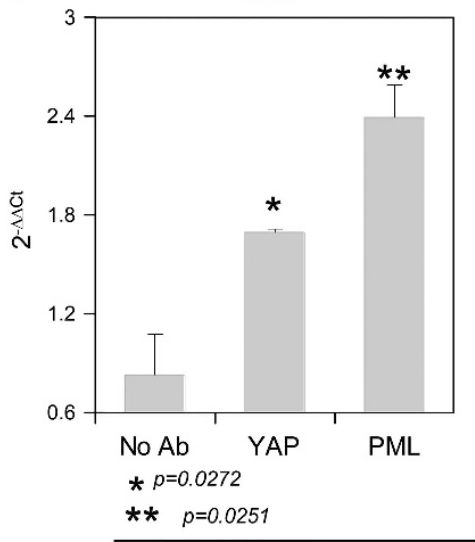

d

Re-ChIP

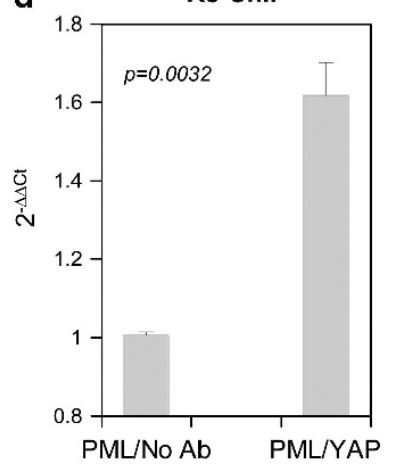

p21 promoter

Figure 4 PML and YAP functionally interact in WRN K/D cells. (a) Western blotting analysis of WCL from HCT116 transfected with siRNA targeting either WRN or GFP (as control) and probed with the indicated antibodies. (b) WCL from HCT116 cells, normalized for YAP expression as indicated in (a), were immunoprecipitated with anti-YAP antibody and IgG as control, respectively. Western blotting analysis was performed with the indicated antibodies. (c and d). ChIP (c) and Re-ChIP (d) assays in shWRNHCT116 cells using the indicated antibodies; YAP and PML proteins are concomitantly recruited on the p21 promoter at the consensus sequence for p53 binding at -2350bp upstream of p21 gene promoter TSS

cardiovascular problems and cancer. Here we document that reduced WRN expression instigates the activation of a functional axis involving YAP, PML and p53, which promote the execution of a senescent program. We also show that YAP and PML signal to p53, which in turn is accumulated and phosphorylated at Ser15 and made transcriptionally active by promoting $p 21^{\text {waf1 }}$ gene expression. Indeed, increased amounts of YAP, PML and p53 proteins are recruited to the regulatory regions of the $p 21^{\text {waf1 }}$ gene, accompanied by augmented local acetylation of histone H4. Although interference of p53 expression does not affect YAP and PML proteins accumulation in WRN-devoid cells, selective knocking down of either YAP or PML expression impairs p53 protein accumulation and phosphorylation and transcriptional activation of the $p 21^{\text {waf } 1}$-target gene. This strongly indicates that YAP and PML act upstream to p53 by converging on oncogenic signals derived from loss of WRN expression (Figure 8d). Previously, we and others reported that YAP, $P M L$ and $p 73$ engage in an auto-regulatory feedback loop axis promoting apoptosis in response to anticancer treatments. ${ }^{10,11,26,27}$ It is well-established that p53 senses DNA damage and activates DNA repair or cell suicide in regard to the amount of damaged DNA. DNA damage activates specific kinases including ATM and p38MAPK, which phosphorylate p53 at Ser15 in response to diverse stress signals. ${ }^{20,21}$ Interestingly, we found that p53 accumulation and Ser15 phosphorylation in response to loss of WRN expression is impaired in either $\mathrm{ATM}^{-1-}$ cells or WRN-deficient cells treated with caffeine. Notably, lack of ATM prevents also YAP and PML protein accumulation. This strongly suggests that $A T M$ is required for enabling the functional axis YAP/PML/p53 to promote senescence upon loss of WRN activity. Indeed, ectopic expression of an ATM kinasedefective mutant in WRN-deficient cells prevents YAP and PML accumulation, as well as p53 stabilization and Ser15 phosphorylation, thereby impairing p $21^{\text {waf1 }}$ induction and the cellular senescence rate. Unlike the effect of the ATM kinasemutant, chemical inhibition of p38MAPK with SB202190 compound affects p53 stabilization and Ser15 phosphorylation, but not YAP and PML accumulation. This implies that loss of WRN expression mimics DNA damage and leads to the activation of signaling pathways governed by specific 

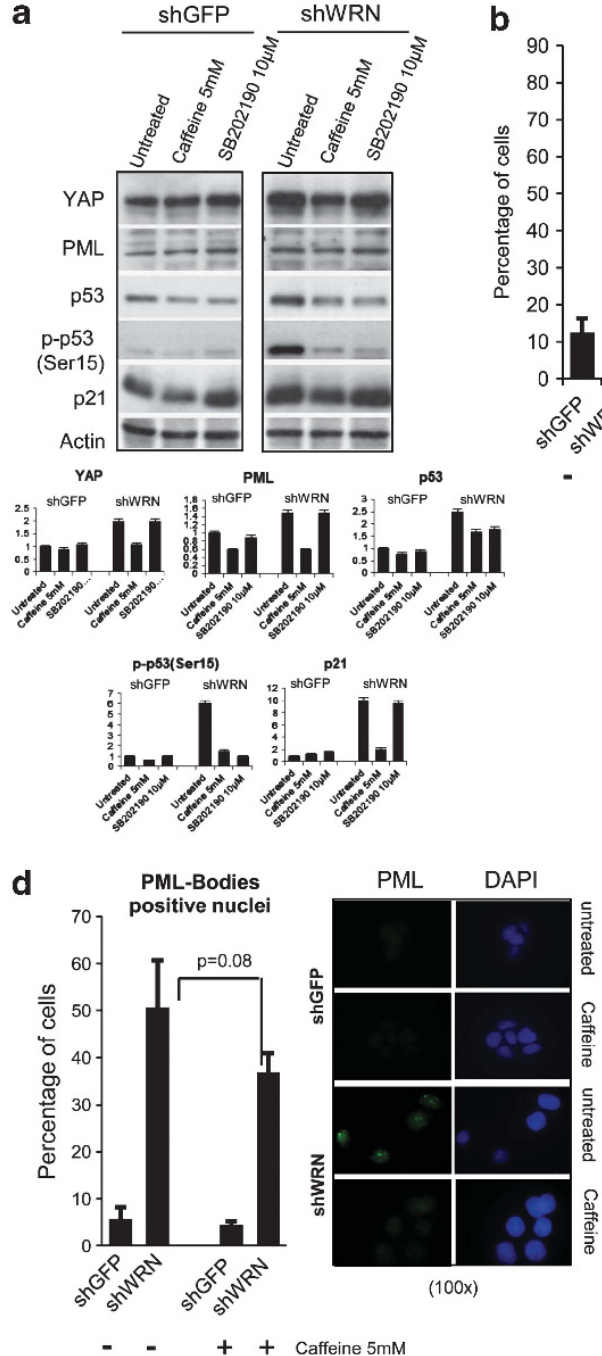
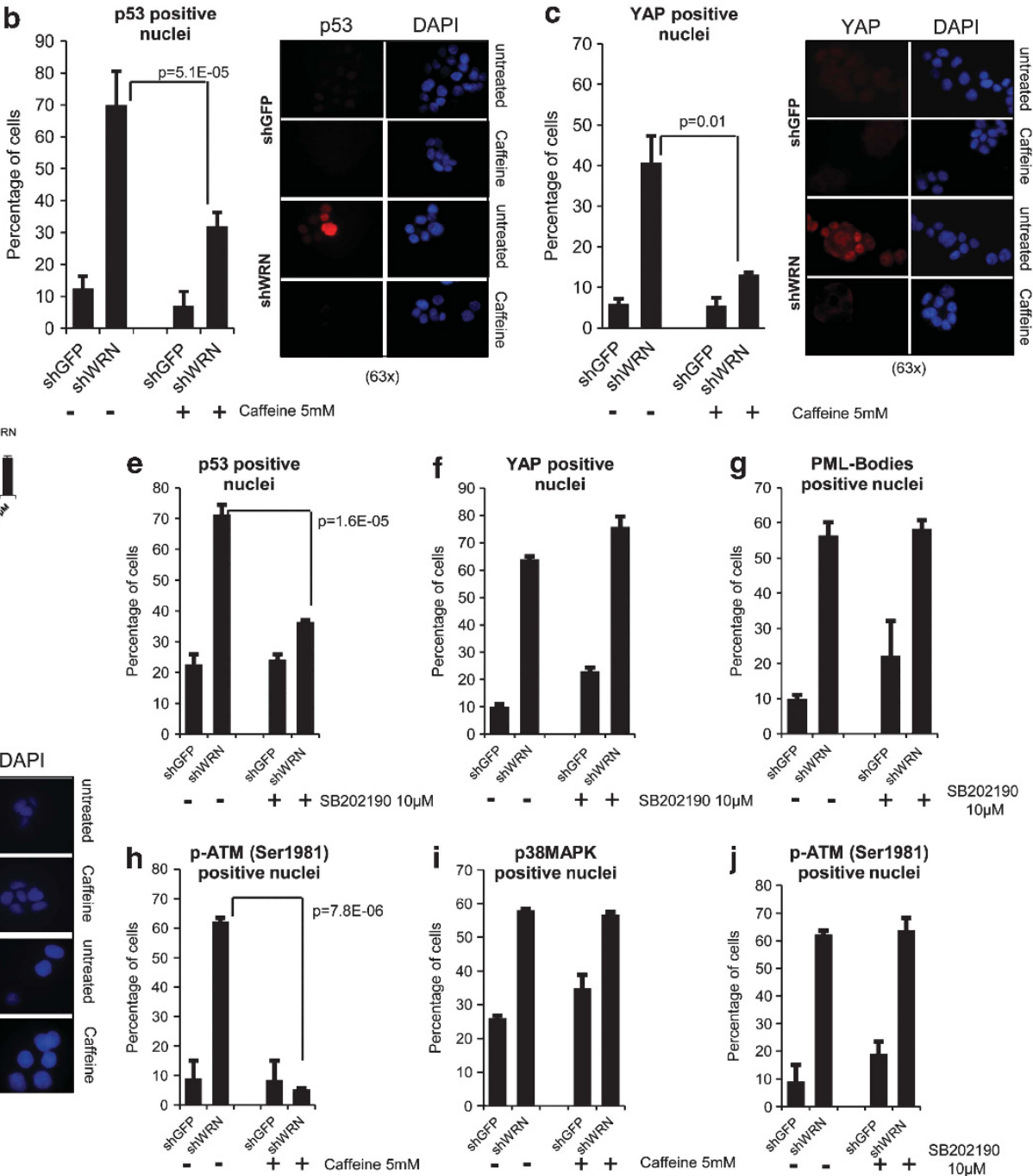

Figure 5 ATM stabilizes YAP in WRN K/D cells. (a) Upper panel: western blotting analysis of WCL from shWRN-HCT116 cells and shGFP-, as control, w/ and w/o Caffeine $5 \mathrm{mM}$ or SB202190 $10 \mu \mathrm{M}$ treatment (3h), and probed with the indicated antibodies. Lower panel: densitometric analysis of the expression of the indicated proteins from cells as from upper panel. Histograms show mean + S.D. values of three independent experiments. (b-d) Left panels: quantitative determination of cells exhibiting p53 stainingpositive nuclei (b), YAP-positive nuclei (c) and PML-Bodies staining-positive nuclei (d) from shWRN-HCT116 cells and shGFP-, as control, w/ and w/o Caffeine $5 \mathrm{mM}$ treatment. Right panels: representative images of cells as from left panels. To better appreciate the effect of caffeine on PML nuclear staining the acquisition of these representative images (d, left panel) was performed at $\times 100$ magnification. (e-g) Quantitative determination of cells exhibiting p53 staining-positive nuclei (e), YAP-positive nuclei (f) and PML-Bodies staining-positive nuclei (g) from shWRN-HCT116 cells and shGFP-, as control, w/ and w/o SB202190 10 $\mu$ M treatment. (h and i). Quantitative determination of cells containing pSer1981-ATM-positive nuclei (h) and p38MAPK-positive nuclei (i) from shWRN-HCT116 cells and shGFP-, as control, w/ and w/o Caffeine $5 \mathrm{mM}$ treatment. (j) Quantitative determination of cells containing pSer1981-ATM-positive nuclei from shWRN-HCT116 cells and shGFP-, as control, w/ and w/o SB202190 $10 \mu \mathrm{M}$ treatment. For all the experiments $(\mathbf{b}-\mathbf{j})$ histograms show mean + S.D. values of three independent experiments

DNA damage-activated kinases, such as ATM and p38MAPK (Figure $8 \mathrm{~d}$ ). We show that the knocking down of p53 expression significantly impairs senescence of human WS-derived fibroblasts by restoring active proliferation. siRNA-mediated interference of either YAP or PML expression increases p21waf1 induction and the fraction of cells re-entering S-phase, albeit to a lesser extent than that of p53. Collectively, our findings point to $\mathrm{p} 53$ as a pivotal protein on which multiple signals derived from loss of WRN function converge, and from which transcriptional programs driving senescence emanate. There is growing evidence that transcriptional coactivators are crucial in imparting target specificity to transcription factors. We have previously shown that YAP drives p73 to apoptotic target genes in response to anticancer treatment. ${ }^{12,13,28}$ Here we show that within the ATM-dependent pathway, YAP, PML and the increased recruitment of the YAP/PML protein complex on the regulatory region of p21 gene signal to p53 and might direct its transcriptional activity toward senescence (Figure 8d).

Growing evidence points at a critical role of YAP in oncogenesis. ${ }^{29-32}$ Indeed, loss of the tumor suppressor activity of the Hippo pathway, as shown in vitro and in vivo models, is determined by a YAP oncogenic switch. ${ }^{33,34}$ In addition, recent work shows that a YAP homolog, TAZ, is highly associated with a mesenchymal (MES) gene expression signature, which features poor overall survival and 
a

H1299

IP :YAP IgG

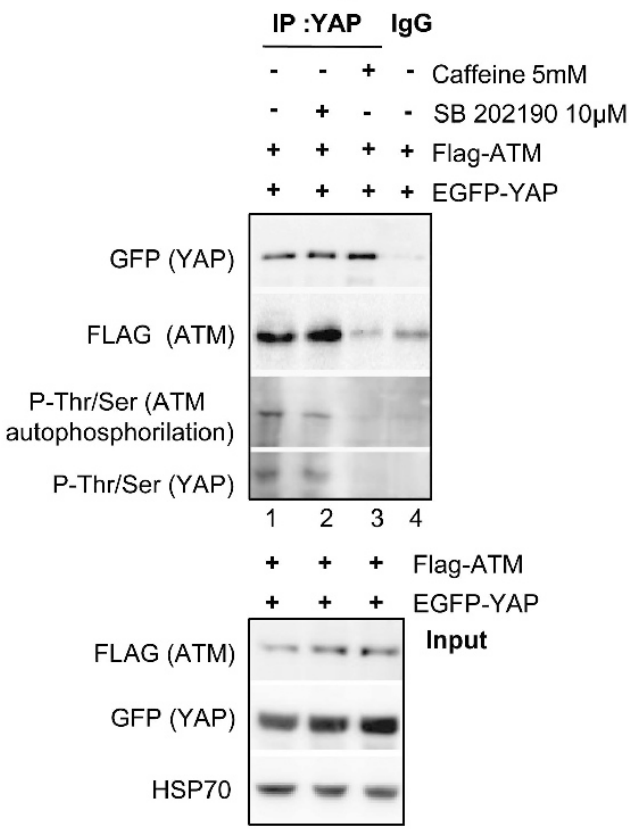

d

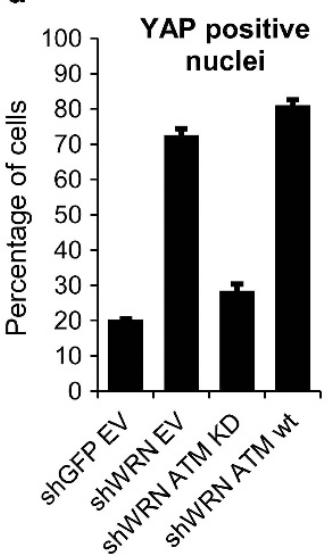

b

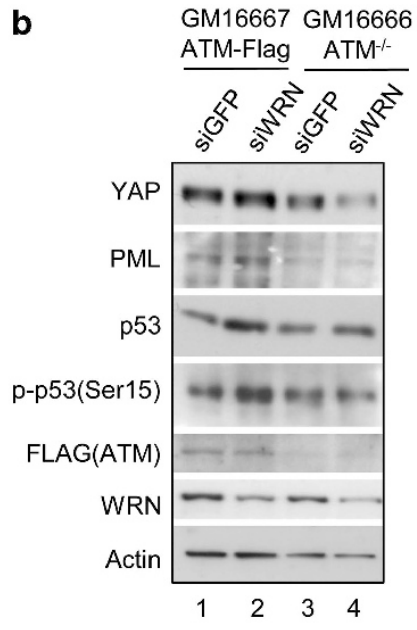

C

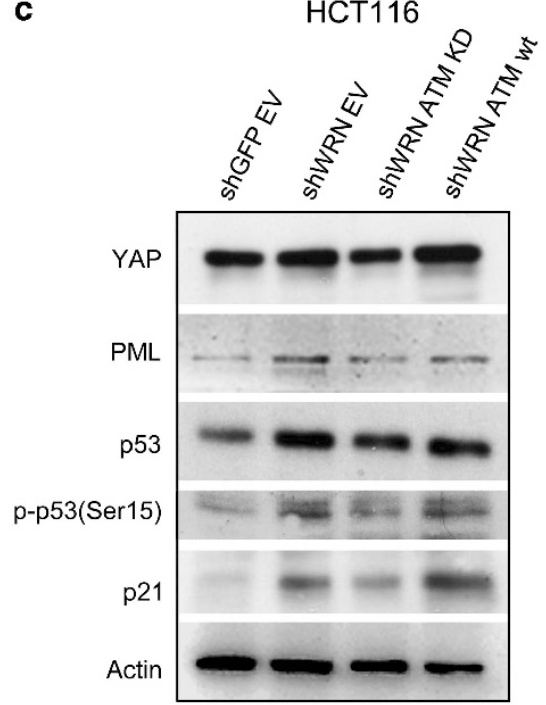

\begin{tabular}{|l|c|c|c|c|}
\hline $\begin{array}{l}\text { YAP folds } \\
\text { over control }\end{array}$ & 1 & 2.5 & 0.9 & 4 \\
\hline $\begin{array}{l}\text { PML folds } \\
\text { over control }\end{array}$ & 1 & 3.2 & 2 & 4.7 \\
\hline $\begin{array}{l}\text { p53 folds } \\
\text { over control }\end{array}$ & 1 & 1.9 & 1.1 & 2.0 \\
\hline $\begin{array}{l}\mathrm{p}-\mathrm{p} 53 \text { (Ser15) } \\
\text { folds } \\
\text { over control }\end{array}$ & 1 & 3 & 1.4 & 4.5 \\
\hline $\begin{array}{l}\mathrm{p} 21 \text { folds } \\
\text { over control }\end{array}$ & 1 & 3.5 & 1.8 & 6.5 \\
\hline
\end{tabular}

Figure 6 ATM binds to and stabilizes YAP by phosphorylating it in WRN K/D, senescent cells. (a) Upper panel: WCL from H1299 cells transfected with pcDNA3Flag-ATM and pEGFP-YAP plasmids and subjected to the indicated treatments, were immunoprecipitated with anti-YAP antibody and IgG as control, respectively. Lower panel: input lysates from $\mathrm{H} 1299$ cells as from upper panels. (b) Western Blotting analysis of WCL from ATM ${ }^{+1+}$ (reconstituted for ATM expression GM16667) and ATM ${ }^{-1-}$ (GM16666) fibroblasts transfected with siRNA against WRN and GFP (as control), and probed with the indicated antibodies. (c) Upper panel: western blotting analysis of WCL from shWRN-HCT116 cells and shGFP-, as control, transiently transfected with either empty (EV), ATM KD (kinase defective) and ATM wt (wild-type) expression vectors, respectively, and probed with the indicated antibodies. Lower panel: densitometric analysis for the expression of the indicated proteins of cells as from upper panel. (d and $\mathbf{e}$ ) Quantitative determination of cells containing YAP-positive nuclei (d) and PML-bodies staining-positive nuclei (e) from cells as from (c). Histograms show mean + S.D. values of three independent experiments. (f) SA- $\beta$-gal staining of cells as from (c). Histograms show mean + S.D. values of three independent experiments. shWRN-HCT116 cells and shGFP-, as control, were collected for protein levels and analyzed for immunofluorescence and SA- $\beta$-Gal staining at $96 \mathrm{~h}$ (4 days) after transfection

resistance to drug treatment in glioblastoma patients. ${ }^{35} \mathrm{TAZ}$ also confers cancer stem cell features to breast cancer cells. ${ }^{36}$ Notably, WS patients develop primarily sarcoma and MES tumors. ${ }^{6}$ This is known to result from a high degree of genomic instability derived from loss of WRN activity. Here we speculate that the accumulation of YAP following loss of WRN is a molecular event that might impinge on the clinical features of WS patients, including their propensity to develop MES-type cancers. ${ }^{6}$ In fact, we show that loss of WRN (hence, YAP upregulation) correlates with increased secretion of protumorigenic and inflammatory cytokines, similarly to what happens to cells acquiring SASP feature when induced by stress stimuli. ${ }^{14,15,23}$ In fact, among the secreted cytokines, we observed IL-6 and IL-8, that we and others have previously shown to be main mediators of the tumorigenicity of breast cancer and malignant mesothelioma cells. $^{24,37}$ In addition, our analysis revealed that several factors not predicted to be secreted, were enriched in the medium conditioned by WRN K/D cells (BCL2, BCL-X, BCL-XL and CASPASE 8). Such factors may be exosome 
a
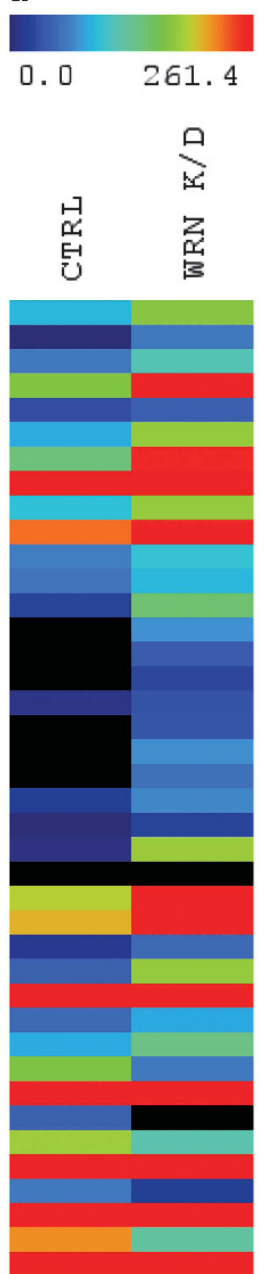

BCL 2a

BCL -6

$\mathrm{BCI}-\mathrm{XL}$

BCL-XL

CASP ASE 8

IP $0-38$

CD 54/ ICAMI

CDg $5 /$ FAS

EGFR

AD AM17

FAS- 1 igand

FGF-1

FGF -2

IFI gamoma

IGFI

ER beta

IL- $121 \mathrm{fa}$

IL-10

IL $-10 R$

IL -17

IL -2

IL -3

IL -30

IL-6

IITH IB III al pha

IP 10/ CRG2

MMP-11/ (Stromelysin-3)

MMP-13/ (coll agenase-3)

MMIP-19

TGF beta3

Amph ire gull in

BCL 10

FLK-1/UEGFR-?

FLT-1/VEGFR-

Glut - cyst ligase

I FIT-al pha

MMPP- 23

MMIP-7/ (Matrilysin)

$\mathrm{MF}-\mathrm{Kb} / \mathrm{p} 65 \mathrm{Re} 1 \mathrm{~A}$

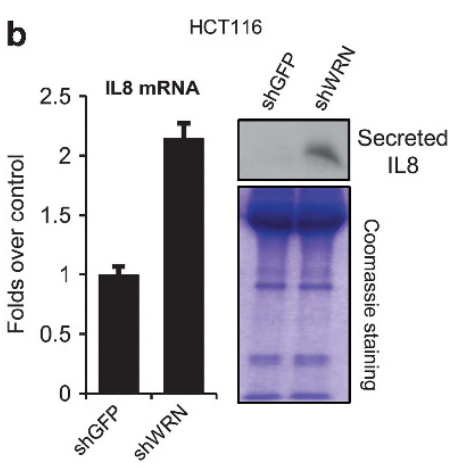

C

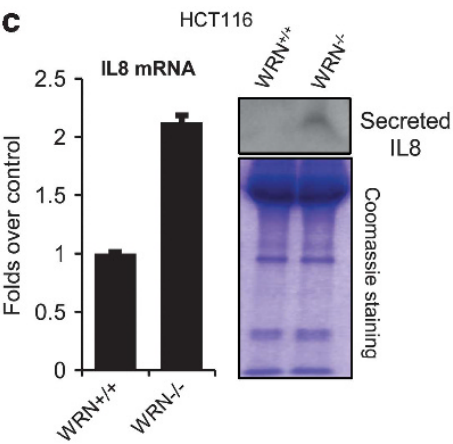

d

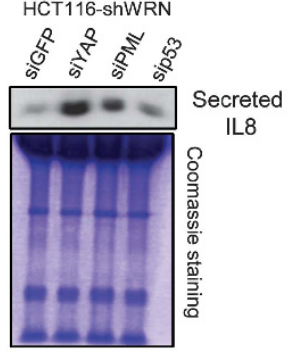

Figure 7 (a) Proteins represented in conditioned media of CTRL and WRN K/D HCT116 cells. Briefly, shWRN-HCT116 cells and shGFP-, as control, were incubated in serum-free medium and media composition was analyzed $24 \mathrm{~h}$ later. (a) Heatmap. The levels of the indicated cytokines, growth factors and receptors are reported as absolute mean value of six replicates. Black boxes indicate ND (not detectable). (b-d) Depletion of WRN increases the secretion of IL-8. (b and $\mathbf{c}$ ) Left panels: Q-PCR analysis of the levels of IL-8 transcript of shWRN-HCT116 cells and shGFP-, as control (b) and of WRN ${ }^{-1-}$ (AG03141) and WRN ${ }^{+/+}$(GM08402) fibroblasts (c). Right panels: western blotting analysis of IL-8 protein levels in conditioned media of shWRN-HCT116 cells and shGFP-, as control (b) and of WRN ${ }^{-1-}$ (AG03141) and WRN ${ }^{+1+}$ (GM08402) (c). For all the experiments histograms show mean + S.D. values of three independent experiments. (d) YAP, PML, P53 modulates IL-8 secretion. Western Blotting analysis of IL-8 protein levels in conditioned media from shWRN-HCT116 cells transfected with the indicated siRNAs. For all the experiments Coomassie staining was used to assess equal loading of the protein gel. Please note that analysis of IL-8 levels was not included in (a)

components, secreted by WRN K/D cells, as this is compatible with the experimental conditions used to perform the analysis. Exosome secretion by tumor cells contributes protumorigenic signals (for example, antiapoptotic signals and immune suppression) to adjacent cells, mediated by the release of intracellular proteins, lipids and nucleic acids into the target cells $^{25}$ (Figure 8d). We therefore speculate that the latter can be an additional mechanism underlying the cancer-prone phenotype of WS patients. In fact, the enrichment for IL-6, IL-8 and for antiapoptotic proteins in the medium conditioned by WRN K/D cells nicely matches the pro-proliferative and clonogenic effects of the CM observed on untransformed and transformed cells, respectively. Further work will address our hypothesis. siRNA-mediated depletion of YAP (and of $\mathrm{PML}$ and $\mathrm{p53}$ ) does apparently exacerbate this phenotype (such as the IL-8 secretion). On the other hand, upregulation of YAP facilitates the loss of WRN-induced senescence (Figure 2). Therefore, we suggest that YAP, within an ATM modulated YAP-PML-p53 functional axis in WS cells acts both by promoting accelerated senescence and by limiting the acquisition of a protumorigenic SASP phenotype. Thus, YAP may exhibit tumor suppression functions into WS cells.

The loss of WRN helicase and its exonuclease activity must have wide ranging effects on the signaling networks of the organism, beyond the immediate lack of an enzymatic function that fine tunes DNA repair, replication and gene transcription. One of the interesting functional links that were unraveled recently for WRN protein is the link with MYC oncogene. ${ }^{38}$ Interestingly, MYC-driven tumorigenesis is inhibited by WS gene deficiency, and this interaction may have direct implications for drug development for both WS and MYC-driven cancers. It would also be interesting to determine 
a

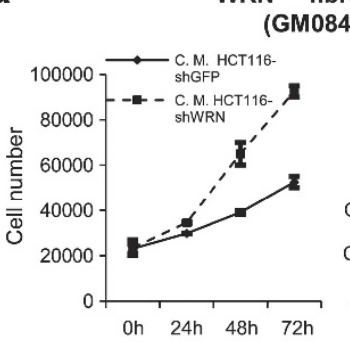

fibroblasts

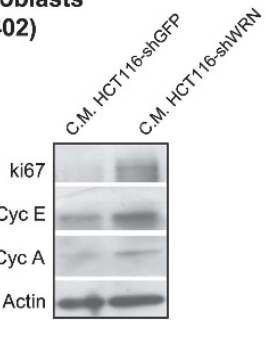

C

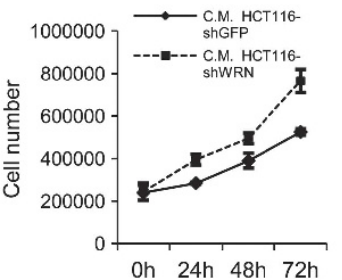

MCF7

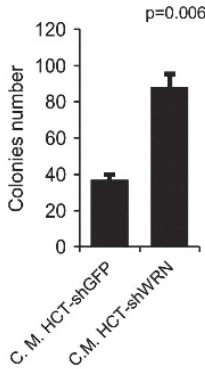

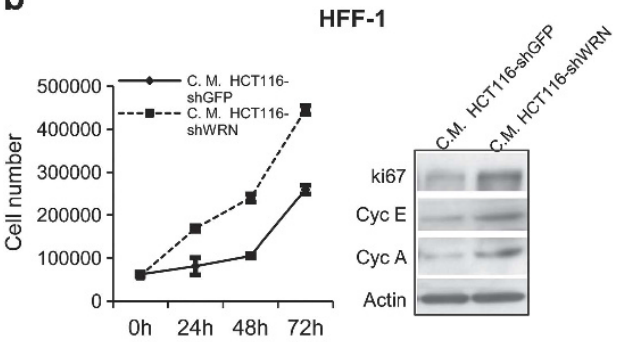

d

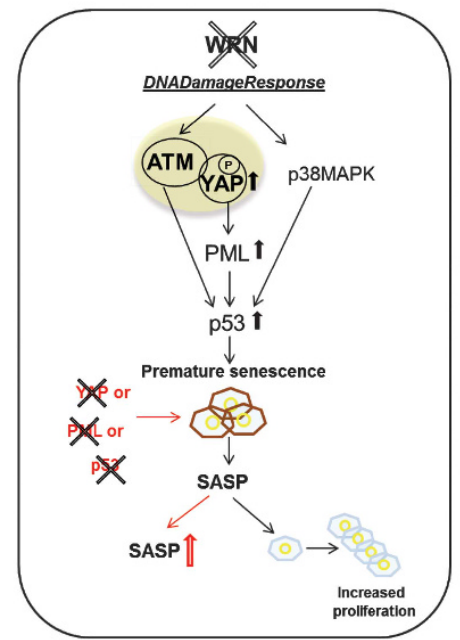

Figure 8 (a-c). CM from WRN ${ }^{-1}$ - cells enhances the protumorigenic properties of multiple cell lines. (a and $\left.\mathbf{b}\right)$ Left panels: proliferation of WRN ${ }^{+/+}(\mathrm{GM} 08402)(\mathbf{a})$ and HFF-1 (b) cells grown in conditioned media derived from shWRN-HCT116 cells and shGFP-, as control. Data show mean + S.D. of three independent cell counts. Right panels: western Blotting analysis of WRN ${ }^{+1+}$ (a) and HFF-1 cells (b), as from left panels, probed with the indicated antibodies (c). Cell growth (left panel) and colony forming ability (right panel) of MCF-7 grown in the same conditioned media as in (a and $\mathbf{b}$ ). For all the experiments histograms show mean + S.D. values of three independent experiments. (d) Working model. Continuous lines indicate the proposed mechanisms as supported by presented data

whether YAP and MYC oncogenes together have a role in WS signaling, as their orthologs in Drosophila fly were shown to be a part of a feedback mechanism that stabilizes tissue growth. ${ }^{39}$ Along this line of evidence, loss of wt-p53 function either by gene deletion or gene mutation could also represent a molecular scenario in which YAP/PML protein complex may signal to oncogenic transcription factors that sustain SASP. Further work is required to elucidate whether accumulation of YAP upon loss of WRN expression may also trigger additional oncogenic mechanisms, linking accelerated senescence to the cancer-prone phenotype of the WS patients.

\section{Materials and methods}

Cell culture and transfection. HCT116, HEK293T, H1299 and MCF-7 cells were cultured in Dulbecco's modified medium (DMEM; Gibco, Invitrogen, Carlsbad, CA, USA) supplemented with 10\% fetal bovine serum (FBS, Gibco), penicillin and streptomycin. For the WRN K/D cells (shWRN-HCT116 and shGFPHCT116 stable cell lines), growth medium was supplemented with 20\% FBS. Primary WS fibroblasts strain AG03141 and primary normal human fibroblasts strain GM08402 (Coriell Institute Biorepository), were cultured in minimum essential medium (GIBCO) with 15\% FBS (Gibco), 2 mM L-Glutamine. Primary ataxia-telangiectasia fibroblasts strain GM16666 $\left(\mathrm{ATM}^{-1-}\right)$ derived from patients and GM16667 (derived from strain GM16666, reconstituted with Flag-ATM expression vector) were a gift from $Y$ Shiloh ${ }^{40}$ and were cultured in DMEM (Gibco) supplemented with $10 \%$ FBS (Gibco), $100 \mu \mathrm{g} / \mathrm{ml}$ hygromycin, penicillin and streptomycin. BJ skin fibroblasts were cultured in minimum essential medium (Gibco) with 10\% FBS (Gibco), $2 \mathrm{mM} \mathrm{L-Glutamine} \mathrm{and} 1 \mathrm{mM}$ sodium pyruvate. HFF-1 (ATCC SCRC-1041) fibroblasts were cultured in DMEM (Gibco) supplemented with $15 \%$ FBS (GIBCO). In all the experiments, cells were transfected with Lipofectamine 2000 (Invitrogen) according to manufacturer's instructions.

Reagents. Caffeine and SB202190 were from Sigma-Aldrich (St. Louis, MO USA). Cells were treated with caffeine $5 \mathrm{mM}$ and SB202190 $10 \mu \mathrm{M}$ for $3 \mathrm{~h}$. CDDP was from Pfizer (New York, NY, USA); DOXO was from Ebewe Pharma (Kundl, Austria) and 5-FU was from Teva (Petach Tikva, Israel). For protein stability assays, cells were treated with $100 \mathrm{mg} / \mathrm{ml}$ cycloheximide (Sigma-Aldrich) at the indicated times. X-Gal for SA- $\beta$-Gal staining was from Invitrogen.

SA- $\beta$-Gal staining. SA- $\beta$-Gal staining was performed on adherent cells according to published protocol. ${ }^{41}$ The percentage of blue SA- $\beta$-Gal-positive cells was determined by counting (at least 500 cells).

BrdU incorporation assay. Brdu incorporation assay was performed using the 5-Bromo-2'-deoxy-uridine Labeling and Detection Kit I (cat no. 11296736001; Roche, Basel, Switzerland) according to manufacturer's protocol. Cells were subjected to $4 \mathrm{~h}$ Brdu pulse. After the immunostaining, Brdu-positive cells were counted under Zeiss Axiovert 200M fluorescence microscope (Zeiss, Oberkochen, Germany), (at least 500 cells).

Plasmids. To generate the stable HCT116-knockdown cell lines, WRN_a siRNA (previously described in Szekely et l. $^{42}$ ) and GFP siRNA oligonucleotides were inserted in pLKO.1-TRC vector ${ }^{43}$ (Addgene, Cambridge, MA, USA). The sequence of the all used siRNA oligonucleotides is available in the Supplementary Informations File. Transfected HCT116 cells were selected with puromycin $(0.5 \mu \mathrm{g} / \mathrm{ml})$ for 3 days. Different clones were derived for both shGFP-HCT116 and shWRNHCT116 by limit dilution of transfected cells (at least two for GFP K/D, named shGFP nos. 1 and 7, and 3 for WRN K/D, named shWRN nos. 8b, 24 and 42). 
Expression vectors for ATM kinase ${ }^{44}$ were purchased from Addgene. Expression vectors for p53 and PML expression were previously described. ${ }^{45}$

Immunoprecipitation and western blotting. Cells were lysed and immunoprecipitated as previously described ${ }^{12}$ using rabbit polyclonal anti-YAP antibody (H-125; Santa Cruz, Santa Cruz, CA, USA) or rabbit polyclonal anti-PML antibody (H238; Santa Cruz) or rabbit serum as control. Western blotting was performed using the following primary antibodies: rabbit polyclonal anti-YAP antibody (H-125; Santa Cruz); mouse monoclonal anti-YAP antibody (no. 56701, Abcam); rabbit polyclonal anti-WRN antibody (H300; Santa Cruz); rabbit polyclonal anti-PML antibody (H238; Santa Cruz); mouse monoclonal anti-p53 antibody (D01; Strano et al. $\left.{ }^{46}\right)$; rabbit polyclonal anti-phosphoSer15 p53 antibody (no. 9284; Cell Signaling, Danvers, MA, USA); rabbit polyclonal anti-p21 antibody (12D1; Cell Signaling); mouse monoclonal anti-Cyclin E antibody (HE12, Santa Cruz); mouse monoclonal anti-Cyclin D1 antibody (AM29; Invitrogen); mouse monoclonal anti-Cyclin A antibody (E23.1; Abcam); rabbit polyclonal anti-Flag (Sigma, no. F7425); mouse monoclonal anti-GFP antibody (sc-9996, B-2; Santa Cruz); rabbit polyclonal anti-phosphoserine/threonine antibody (ab 17464; Abcam); rabbit polyclonal anti-Ki-67 antibody (ab15580; Abcam); mouse monoclonal anti-Actin antibody (AC74; Sigma-Aldrich). Western blot analysis was performed with the aid of the enhanced chemiluminescence system (Amersham Pharmacia Biotech, Piscataway, NJ, USA).

Immunofluorescence. Human HCT116 stable cell lines (shWRN-HCT116 and shGFP-HCT116) were grown on coverslips and subjected to the indicated treatments, were fixed in 4\% PBS-paraformaldeyde for $15 \mathrm{~min}$; incubated in $0.5 \%$ TritonX-100 for $10 \mathrm{~min}$ and then blocked with $5 \%$ BSA in PBS for $1 \mathrm{~h}$. Coverslips were incubated $4{ }^{\circ} \mathrm{C}$ overnight with primary antibodies in humidified chamber. Mouse monoclonal anti-YAP antibody (Abcam, no. 56701), rabbit polyclonal anti-PML antibody (H238; Santa Cruz); monoclonal anti-p53 antibody $\left(\mathrm{DO}^{46}\right)$, mouse monoclonal anti-phosphoSer1981 ATM (10H11.E12; Cell Signaling) and anti-p38 MAP Kinase (Cell Signaling; no. 9212) were used. Coverslips were then washed three times in PBS and then incubated with the following secondary antibodies for $1 \mathrm{~h}$ : goat anti-rabbit Alexa Fluor 488 (A11034; Invitrogen) and goat anti mouse Alexa Fluor 594 (A11032; Invitrogen). After washing, the nuclei were counterstained with DAPI. Cells were then washed twice with PBS and observed under Zeiss Axiovert 200M fluorescence microscope. Specific fields were photographed with a digital camera equipped with Zeiss Axiovision acquisition software. The percentage of positive cells was determined by counting (at least 500 cells).

Formaldehyde crosslinking, chromatin immunoprecipitation and Re-Chlp. Chromatin immunoprecipitation experiments were performed on HCT116 stable cell lines (shWRN-HCT116 and shGFP-HCT116) according to protocol previously described. ${ }^{12}$ The following antibodies were used: WRN (H300; Santa Cruz); YAP (H-125; Santa Cruz); PML (H238; Santa Cruz); p53 (Ab7; Calbiochem); and H4ac (06-866; Millipore Upstate). Enrichment in p21 promoter was evaluated using PCR and RTQ-PCR.

Fold enrichment method: ${ }^{47}$ the ChIP signals are divided by the no-antibody signals, representing the ChIP signal as the fold increase in signal relative to the background signal.

For the Re-ChIP experiments performed on stable cell lines shWRN-HCT116 the chromatin was eluted with $10 \mathrm{mM}$ DTT at room temperature for $30 \mathrm{~min}$. The eluted chromatin was diluted 10 -fold with re-chIP buffer ( $15 \mathrm{mM}$ Tris-Cl pH 7.5, $1 \%$ Triton $\mathrm{X}-100,150 \mathrm{mM} \mathrm{NaCl}$ and $1 \mathrm{mM} \mathrm{EDTA}$ ) and the second round of ChIP was carried out according to the regular ChIP procedure. The p21 promoter occupancy was analyzed by qRT-PCR. Primers used for the amplification of p21 region were listed in the Supplementary Informations file. $P$-values were calculated with two-tailed $t$-test. Normalization was performed to the amount of input chromatin. Statistically significant results were referred with $P$-value $<0.05$.

The consensus sequence for $p 53$ binding at $-2350 \mathrm{bp}$ upstream of p21 gene promoter TSS was amplified for both assays. This core sequence was also reported in. ${ }^{48}$

RNA interference. To knockdown WRN protein expression, two different oligonucleotide sequences were used. The sequences were previously described in Szekely et $\mathrm{A}^{42}$ and Turaga et al. ${ }^{49}$ (HSS111385).

HCT116, shWRN-HCT116 (and control shGFP-) cells, primary normal (GM08402) and WS fibroblasts (AG03141) were transiently transfected with either
$2 \mu \mathrm{g}$ of the control siRNAs (GFP) or siWRN, siYAP and siPML or sip53 in $60 \mathrm{~mm}$ dish. Cells were harvested at different time points. The effects of YAP or PML or p53 interference on stable shWRN-HCT116 were evaluated 4 days (96 h) after siRNA transfection. The effects of YAP or PML or p53 interference on WS (AG03141) cells were evaluated about 8 days $(192 \mathrm{~h})$ after siRNA transfection. All siRNA oligonucleotide sequences are available in the Supplementary informations file.

RNA extraction, reverse transcriptase reaction and RTQ-PCR. Cells were harvested in TRIZOL reagent (Invitrogen) and total RNA was isolated according to the manufacturer's instructions. Five micrograms of total RNA was reverse-transcribed at $37^{\circ} \mathrm{C}$ for $45 \mathrm{~min}$ in the presence of random hexamers and Moloney murine leukemia virus reverse transcriptase (Invitrogen). RTQ-PCR analysis was carried out using specific oligonucleotides for the indicated genes. The sequence of the used oligonucleotides is available in the Supplementary informations file. PCR amplification was carried out using Sybr Green PCR Master Mix (Applied Biosystem, Foster City, CA, USA) and specific primers for GAPDH and/or ACTIN, YAP, PML and p53 transcripts.

Polysome fractionation and RNA extraction by sucrose gradients. The polysome fractionation was performed as previously described. $^{50}$

We generated two pools of RNA: (1) polysomal RNAs constituted by pooling aliquots from the fractions containing two or more ribosomes as determined from the UV profiles and (2) non polysomal RNAs constituted by pooling the remaining aliquots. The total mRNAs from unfractionated cell lysates were also isolated to check the steady-state levels. TRIZOL (Gibco) was used to extract RNAs. Doublestranded CDNA was prepared using Superscript II and random hexamer primers (Invitrogen). The same cDNA preps were used for real-time PCR. PCR amplification was carried out using SYBR Green PCR Master Mix (Applied Biosystem) and specific primers for GAPDH, YAP, PML and p53 transcripts. The values shown in the figures are the $2^{-\triangle \Delta C T}$ of each mRNA after normalization with GAPDH from triplicates. The levels of YAP, PML and p53 transcripts by polysomal fractions were also evaluated considering their total mRNA extracted by the input obtaining the same result.

Cytokine antibody microarray. For the cytokine expression profile we used the array by Spring Bioscience (Pleasenton, CA, USA) following the manufacturer's instructions (ACK-100). Conditioned media from HCT116 cells stably knockeddown for WRN (shWRN-HCT116) and for GFP (shGFP-HCT116) incubated in serum-free medium for $24 \mathrm{~h}$ were analyzed. Each slide (containing six replicates) hybridized and Cy3 fluorescence acquired by microarray scanner with a scan resolution of $10 \mu \mathrm{m}$ (Agilent Technologies, Santa Clara, CA, USA). The images were quantified using Agilent Feature Extraction (AFE) software (Agilent Technologies).

Statistical methods and Pathway analyses. Statistical analysis was done using two-sided Student's $t$-test. Differences were deemed statistically significant at $P<0.05$.

Colony formation assay. MCF-7 cells $\left(1 \times 10^{3}\right)$ were seeded in $35-\mathrm{mm}$ dishes and grown in $\mathrm{CM}$ (CM as reported in the figure) derived from stable cell line shWRN-HCT116 or shGFP-HCT116, as control, for about 10 days. Cells were then stained with crystal violet and colonies counted.

\section{Conflict of Interest}

The authors declare no conflict of interest.

Acknowledgements. We thank Virginia Mazack for valuable comments on the first version of the manuscript. This work was supported by AIRC (no. 10454 to G B and no. 5954 to SS), by PA Breast Cancer Coalition Grants (no. 60707 and no. 920093) plus the Geisinger Clinic (to MS).

1. Bachrati CZ, Hickson ID. RecQ helicases: guardian angels of the DNA replication fork. Chromosoma 2008; 117: 219-233.

2. Hanada K, Hickson ID. Molecular genetics of RecQ helicase disorders. Cell Mol Life Sci 2007; 64: 2306-2322. 
3. Sharma S, Doherty KM, Brosh RM Jr. Mechanisms of RecQ helicases in pathways of DNA metabolism and maintenance of genomic stability. Biochem J 2006; 398: 319-337.

4. Soultanas P, Wigley DB. Unwinding the 'Gordian knot' of helicase action. Trends Biochem Sci 2001; 26: 47-54.

5. Yu CE, Oshima J, Fu YH, Wijsman EM, Hisama F, Alisch R et al. Positional cloning of the Werner's syndrome gene. Science 1996; 272: 258-262.

6. Goto M, Miller RW, Ishikawa Y, Sugano H. Excess of rare cancers in Werner syndrome (adult progeria). Cancer Epidemiol Biomarkers Prev 1996; 5: 239-246.

7. Hickson ID. RecQ helicases: caretakers of the genome. Nature reviews. Cancer 2003; 3: $169-178$.

8. Opresko PL. Telomere ResQue and preservation-roles for the Werner syndrome protein and other RecQ helicases. Mec Ageing Dev 2008; 129: 79-90.

9. Sudol M, Bork P, Einbond A, Kastury K, Druck T, Negrini M et al. Characterization of the mammalian YAP (Yes-associated protein) gene and its role in defining a novel protein module, the WW domain. J Biol Chem 1995; 270: 14733-14741.

10. Bertini E, Oka T, Sudol M, Strano S, Blandino G. YAP: at the crossroad between transformation and tumor suppression. Cell Cycle 2009; 8: 49-57.

11. Strano S, Fausti F, Di Agostino S, Sudol M, Blandino G. PML. Surfs into HIPPO Tumor Suppressor Pathway. Frontiers Oncol 2013; 3: 36.

12. Lapi E, Di Agostino S, Donzelli S, Gal H, Domany E, Rechavi G et al. PML, YAP, and p73 are components of a proapoptotic autoregulatory feedback loop. Mol Cell 2008; 32 803-814.

13. Strano S, Monti O, Pediconi N, Baccarini A, Fontemaggi G, Lapi E et al. The transcriptional coactivator Yes-associated protein drives p73 gene-target specificity in response to DNA Damage. Mol Cell 2005; 18: 447-459.

14. Coppe JP, Desprez PY, Krtolica A, Campisi J. The senescence-associated secretory phenotype: the dark side of tumor suppression. Annu Rev Pathol 2010; 5 : 99-118.

15. Coppe JP, Patil CK, Rodier F, Sun Y, Munoz DP, Goldstein J et al. Senescence-associated secretory phenotypes reveal cell-nonautonomous functions of oncogenic RAS and the p53 tumor suppressor. PLOS Biol 2008; 6: 2853-2868.

16. Sliwinska MA, Mosieniak G, Wolanin K, Babik A, Piwocka K, Magalska A et al. Induction of senescence with doxorubicin leads to increased genomic instability of HCT116 cells. Mec Ageing Dev 2009; 130: 24-32.

17. te Poele RH, Okorokov AL, Jardine L, Cummings J, Joel SP. DNA damage is able to induce senescence in tumor cells in vitro and in vivo. Cancer Res 2002; 62: 1876-1883.

18. Pearson M, Carbone R, Sebastiani C, Cioce M, Fagioli M, Saito S et al. PML regulates p53 acetylation and premature senescence induced by oncogenic Ras. Nature 2000; 406 207-210.

19. Oka T, Mazack V, Sudol M. Mst2 and Lats kinases regulate apoptotic function of Yes kinase-associated protein (YAP). J Biol Chem 2008; 283: 27534-27546.

20. Banin S, Moyal L, Shieh S, Taya Y, Anderson CW, Chessa L et al. Enhanced phosphorylation of p53 by ATM in response to DNA damage. Science 1998; 281 : 1674-1677.

21. She QB, Chen N, Dong Z. ERKs and p38 kinase phosphorylate p53 protein at serine 15 in response to UV radiation. J Biol Chem 2000; 275: 20444-20449.

22. Sarkaria JN, Busby EC, Tibbetts RS, Roos P, Taya Y, Karnitz LM et al. Inhibition of ATM and ATR kinase activities by the radiosensitizing agent, caffeine. Cancer Res 1999; 59: 4375-4382.

23. Basu D, Reyes-Mugica M, Rebbaa A. Role of the Beta catenin destruction complex in mediating chemotherapy-induced senescence-associated secretory phenotype. PloS One 2012; 7: e52188.

24. Canino C, Mori F, Cambria A, Diamantini A, Germoni S, Alessandrini G et al. SASP mediates chemoresistance and tumor-initiating-activity of mesothelioma cells. Oncogene 2011; 31: 3148-3163.

25. Yang $\mathrm{C}$, Robbins PD. The roles of tumor-derived exosomes in cancer pathogenesis. Clin Dev Immunol 2011; 2011: 842849

26. Strano S, Munarriz E, Rossi M, Castagnoli L, Shaul Y, Sacchi A et al. Physical interaction with Yes-associated protein enhances p73 transcriptional activity. J Biol Chem 2001; 276: 15164-15173.

27. Yuan M, Tomlinson V, Lara R, Holliday D, Chelala C, Harada T et al. Yes-associated protein (YAP) functions as a tumor suppressor in breast. Cell Death Differ 2008; 15: 1752-1759.
28. Cioce M, Blandino G. PGC1alpha confers specificity-metabolic stress and p53-dependent transcription. Mol Cell 2011; 44: 515-516.

29. Barry ER, Morikawa T, Butler BL, Shrestha K, de la Rosa R, Yan KS et al. Restriction of intestinal stem cell expansion and the regenerative response by YAP. Nature 2013; 493: 106-110.

30. Zender L, Spector MS, Xue W, Flemming P, Cordon-Cardo C, Silke J et al. Identification and validation of oncogenes in liver cancer using an integrative oncogenomic approach. Cell 2006; 125: 1253-1267.

31. Zhao B, Kim J, Ye X, Lai ZC, Guan KL. Both TEAD-binding and WW domains are required for the growth stimulation and oncogenic transformation activity of yes-associated protein. Cancer Res 2009; 69: 1089-1098.

32. Zhao B, Ye X, Yu J, Li L, Li W, Li S et al. TEAD mediates YAP-dependent gene induction and growth control. Genes Dev 2008; 22: 1962-1971.

33. Zhao B, Li L, Lei Q, Guan KL. The Hippo-YAP pathway in organ size control and tumorigenesis: an updated version. Genes Dev 2010; 24: 862-874.

34. Zhao B, Li L, Wang L, Wang CY, Yu J, Guan KL. Cell detachment activates the Hippo pathway via cytoskeleton reorganization to induce anoikis. Genes Dev 2012; 26: 54-68.

35. Bhat KP, Salazar KL, Balasubramaniyan V, Wani K, Heathcock L, Hollingsworth F et al. The transcriptional coactivator TAZ regulates mesenchymal differentiation in malignant glioma. Genes Dev 2011; 25: 2594-2609.

36. Cordenonsi M, Zanconato F, Azzolin L, Forcato M, Rosato A, Frasson C et al. The Hippo transducer TAZ confers cancer stem cell-related traits on breast cancer cells. Cell 2011; 147: 759-772.

37. Sansone P, Storci G, Tavolari S, Guarnieri T, Giovannini C, Taffurelli M et al. IL-6 triggers malignant features in mammospheres from human ductal breast carcinoma and normal mammary gland. J Clin Invest 2007; 117: 3988-4002.

38. Moser R, Toyoshima M, Robinson K, Gurley KE, Howie HL, Davison J et al. MYC-driven tumorigenesis is inhibited by WRN syndrome gene deficiency. Mol Cancer Res 2012; 10: $535-545$

39. Neto-Silva RM, de Beco S, Johnston LA. Evidence for a growth-stabilizing regulatory feedback mechanism between Myc and Yorkie, the Drosophila homolog of Yap. Dev Cell 2010; 19: 507-520

40. Ziv Y, Bar-Shira A, Pecker I, Russell P, Jorgensen TJ, Tsarfati I et al. Recombinant ATM protein complements the cellular A-T phenotype. Oncogene 1997; 15: 159-167.

41. Dimri GP, Lee X, Basile G, Acosta M, Scott G, Roskelley C et al. A biomarker that identifies senescent human cells in culture and in aging skin in vivo. Proc Natl Acad Sci USA 1995; 92: 9363-9367.

42. Szekely AM, Bleichert F, Numann A, Van Komen S, Manasanch E, Ben Nasr A et al. Werner protein protects nonproliferating cells from oxidative DNA damage. Mol Cell Biol 2005; 25: 10492-10506.

43. Moffat J, Grueneberg DA, Yang X, Kim SY, Kloepfer AM, Hinkle G et al. A lentiviral RNAi library for human and mouse genes applied to an arrayed viral high-content screen. Cell 2006; 124: 1283-1298.

44. Canman CE, Lim DS, Cimprich KA, Taya Y, Tamai K, Sakaguchi K et al. Activation of the ATM kinase by ionizing radiation and phosphorylation of p53. Science 1998; 281 : 1677-1679.

45. Bernardi R, Scaglioni PP, Bergmann S, Horn HF, Vousden KH, Pandolfi PP. PML regulates p53 stability by sequestering Mdm2 to the nucleolus. Nature Cell Biol 2004; 6 : 665-672.

46. Strano S, Munarriz E, Rossi M, Cristofanelli B, Shaul Y, Castagnoli $L$ et al. Physical and functional interaction between p53 mutants and different isoforms of p73. J Biol Chem 2000; 275: 29503-29512.

47. Winter N, Nimzyk R, Bosche C, Meyer A, Bullerdiek J. Chromatin immunoprecipitation to analyze DNA binding sites of HMGA2. PloS One 2011; 6: e18837.

48. Vigneron AM, Vousden $\mathrm{KH}$. An indirect role for ASPP1 in limiting p53-dependent p21 expression and cellular senescence. EMBO J 2012; 31: 471-480.

49. Turaga RV, Paquet ER, Sild M, Vignard J, Garand C, Johnson FB et al. The Werner syndrome protein affects the expression of genes involved in adipogenesis and inflammation in addition to cell cycle and DNA damage responses. Cell Cycle 2009; 8: 2080-2092.

50. Paronetto MP, Messina V, Bianchi E, Barchi M, Vogel G, Moretti C et al. Sam68 regulates translation of target mRNAs in male germ cells, necessary for mouse spermatogenesis. J Cell Biol 2009; 185: 235-249.

\section{Supplementary Information accompanies this paper on Cell Death and Differentiation website (http://www.nature.com/cdd)}

